\title{
Coma anóxico-isquémico en 46 pacientes. Evolución a treinta días y su relación con los reflejos de tronco
}

\author{
Luis Lay-Son $\mathbf{R}^{1,2}$, Patricio Varas $\mathbf{F}^{\mathbf{1}}$. \\ The absence of brainstem reflexes \\ does not predict short term mortality \\ in anoxic ischemic coma
}

Background: Anoxic-ischemic coma has a poor outcome with a high rate of mortality and morbidity. Therefore, clinical predictors of prognosis are needed for therapeutic decision-making. Patients and methods: Prospective analysis of 46 patients, 31 male, age range 19-85 years, with anoxic-ischemic coma following cardiac arrest. All the patients included in our study remained comatose with a Glasgow Coma Scale (GCS) score of six or less points, after their stabilization in the Intensive Care Unit. They were evaluated clinically using the pupillary light reflex, corneal reflex and vestibulo-ocular reflex testing, induced by caloric stimulation with cold water. Survival was evaluated using life tables. All patients were followed until the thirtieth day after the anoxic-ischemic event. Results: Thirty five patients (76\%) died within the next twenty-nine days, 8 patients (18\%) reached the vegetative state, 2 patients (4\%) achieved a recovery with disability, and only 1 patient (2\%) was discharged without sequelae. One day, five and 30 days survival rates were 89, 53 and $29 \%$, respectively. The abolition of all brainstem reflexes was not a predictor of mortality. Conclusion: Thirty day survival in this group of patients was $29 \%$ and the absence of brainstem reflexes was not a predictor of mortality (Rev Méd Chile 2006; 134: 441-6).

(Key words: Brain ischemia; Brain stem; Coma; Hypoxia, brain)

Recibido el 13 de junio, 2005. Aceptado el 12 de septiembre, 2005.

${ }^{1}$ Hospital Barros Luco-Trudeau, Universidad de Santiago de Chile. ${ }^{2}$ Hospital Parroquial de San Bernardo, Santiago de Chile.

$\mathrm{E}^{1}$ coma anóxico-isquémico posterior a un paro cardiorrespiratorio (PCR) de causa médica tiene peor pronóstico en comparación con el

Correspondencia a: Dr. Luis Lay-Son R. San Francisco 3097, San Miguel, Santiago-Chile. Fonos: 2-3948941-3948942. Fax: 2-3948941. E mail: llay-son@usach.cl coma de origen traumático o secundario a un traumatismo encéfalo craneal ${ }^{1-3}$. La aplicación de técnicas de resucitación cardiopulmonar (RCP) y la optimización del manejo de los pacientes en Unidades de Cuidados Intensivos (UCI), ha permitido rescatar pacientes que han sufrido PCR. Sin embargo, se mantiene una alta tasa de mortalidad, que oscila entre 50 y $90 \%$, provocada en las 
primeras semanas por complicaciones pulmonares o cardíacas, incluso en pacientes que han sufrido PCR intrahospitalario o que han sido tratados en una UCI ${ }^{4-7}$. En cuanto a los casos que sobreviven, la evolución también es poco favorable, pues se asocia a un elevado índice de discapacidad que les impide reanudar su vida en las condiciones previas; menos de $20 \%$ logra un grado de recuperación similar a su estado basal y alrededor de $55 \%$ de éstos quedan con una discapacidad severa, aun después de ser sometidos a terapia de rehabilitación integral ${ }^{8,9}$. La determinación precoz del desenlace posterior a la RCP es de suma relevancia, atendiendo los aspectos médicos, éticos, legales económicos y sociales derivados de esta situación, que afectan al paciente y su familia por la incertidumbre de la recuperación, al equipo médico por la dedicación requerida y al servicio de salud por el alto costo implicado en la atención. De ahí que se ha intentado -sin éxitodiseñar un método útil para predecir con exactitud el pronóstico de estos pacientes mediante estudios clínicos, electrofisiológicos, imagenológicos, y de variables de laboratorio ${ }^{10-15}$.

El presente trabajo tuvo dos objetivos: primero obtener una experiencia local en cuanto a la calidad de supervivencia en pacientes que han sufrido un coma anóxico-isquémico y, segundo, evaluar si la ausencia total de los reflejos troncoencefálicos durante la evolución se comportaba como un parámetro determinante de desenlace fatal al cabo de los treinta días.

\section{PACIENTES y MÉTodo}

Este estudio se realizó en los hospitales Barros Luco-Trudeau y Parroquial de San Bernardo, ubicados en el área sur de Santiago, con un protocolo prospectivo que contempló los siguientes criterios de inclusión: pacientes que sufrieron un PCR de causa general sistémica, no neurológica ni traumática, que luego de ser recuperados mediante maniobras de RCP lograron estabilización hemodinámica y permanecieron al menos durante $4 \mathrm{~h}$ en coma, con un puntaje igual o menor de 6 en la Escala de Glasgow (EG) ${ }^{16}$. Se usó esta escala por su uso masivo y menor variabilidad interobservador, y este puntaje como línea de corte puesto que refleja un grado de depresión de conciencia comparable al estado de coma. Los pacientes seleccionados fueron sometidos a evaluación de los reflejos del tronco encefálico: fotomotor, corneal y vestíbulo-ocular inducido mediante estimulación calórica laberíntica con agua fría, unilateral, alternada. Se privilegió este método de evaluación dada su mayor precisión en relación a los movimientos oculocefáli$\cos ^{2}$. A todos los pacientes se les efectuó un seguimiento en los 30 días posteriores al PCR para observar el estado neurológico alcanzado y se determinó una curva de sobreviva de acuerdo al método de Kaplan-Meier.

\section{Resultados}

Un total de 46 pacientes cumplieron con los requisitos estipulados y se les analizó las variables generales: edad, sexo, patología de base, causa y lugar del PCR y las variables específicas, que eran los reflejos de tronco. El grupo se distribuyó de acuerdo al sexo en 31 hombres (67\%) y 15 mujeres (33\%), con una mediana de 56,5 años (rango 19-85). Según el sitio de ocurrencia del evento, $70 \%(n=32)$ de los pacientes presentaron el PCR al interior del recinto asistencial. Las patologías que originaron el PCR se detallan en la Tabla 1, apreciándose que $70 \%$ correspondió a causas cardíacas y respiratorias. En cuanto al momento en que se les realizó la evaluación clínica, en $54 \%$ de los pacientes acaeció dentro de las 24 h, en $83 \%$ a las 48 h, en $91 \%$ antes de las 72

\section{Tabla 1. Etiología del paro cardiorrespiratorio (PCR)} en 46 pacientes

\begin{tabular}{|lrc|}
\hline Patología subyacente & \multicolumn{3}{c|}{ Número de pacientes } \\
& $\mathrm{n}$ & $\%$ \\
\hline Falla cardíaca & 21 & $(46)$ \\
Falla respiratoria & 11 & $(24)$ \\
Sepsis & 5 & $(11)$ \\
Falla multisistémica & 2 & \\
Coma hiperglicémico & 2 & \\
Intoxicación por organofosforados & 2 & \\
Coma hipoglicémico & 1 & \\
Intoxicación por amitriptilina & 1 & \\
Accidente anestésico & 1 & \\
\hline
\end{tabular}


h y en el $100 \%$ al cabo de las siguientes 120 h. El análisis clínico reveló la siguiente distribución de acuerdo al puntaje en la EG: 3 puntos: $25 \%$; 4 puntos: $41 \%$; 5 puntos: $23 \%$ y 6 puntos: $11 \%$. De acuerdo a la evaluación de los reflejos de tronco encefálico, $75 \%$ tenía reflejo fotomotor presente,

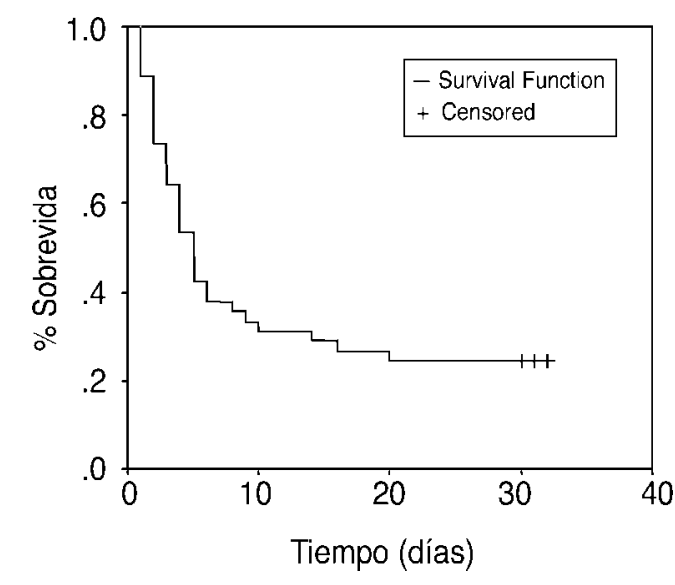

Figura 1. Curva de supervivencia global. La posibilidad de sobrevivir más allá de 30 días es 28,8\%.

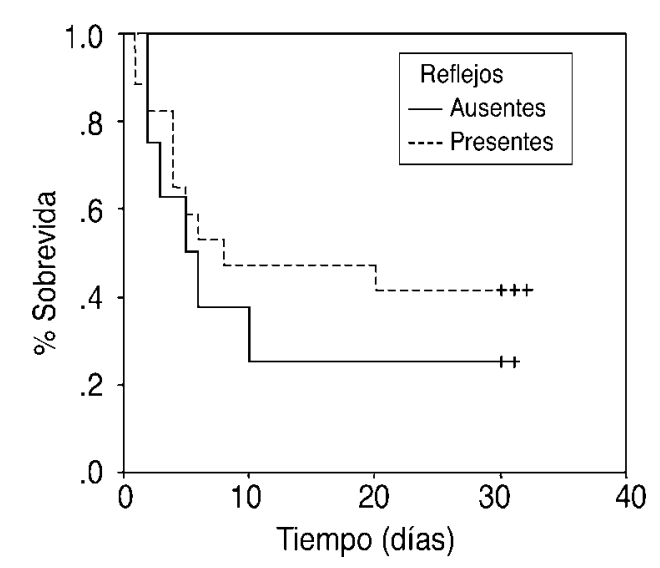

Figura 2. Curva de supervivencia según la presencia o ausencia de los reflejos de tronco: La comparación de las curvas de sobrevida mediante el test Log-Rank, arroja un valor $p=0,4566$ : no hubo diferencias significativas en la distribución de la sobrevida entre ambos grupos.
$41 \%$ reflejo corneal y $62 \%$ tenía respuesta vestíbulo ocular conservada. Al cabo de 30 días de ocurrido el PCR, hallamos una mortalidad global de $76 \% \quad(n=35)$ y una supervivencia de $24 \%$ $(\mathrm{n}=11)$. De los sobrevivientes, 8 pacientes quedaron en estado vegetativo (EV), 2 fueron dados de alta con algún grado de secuelas y solamente 1 paciente egresó con recuperación ad integrum. La mortalidad global de acuerdo al puntaje en la EG mostró una diferencia entre los puntajes extremos de 3 y 6 puntos. Pacientes con puntaje de 3 puntos tuvieron $91 \%$ de mortalidad, con 4 puntos $61 \%$, con 5 puntos $90 \%$ y con 6 puntos $60 \%$. La mortalidad acumulada global mostró que el desenlace fatal aconteció en 50\% de los pacientes al cabo de los tres primeros días y en más de $75 \%$ en los primeros siete días. Al analizar la curva (Kaplan-Meier) de supervivencia global que incluye a todos los pacientes, se observa que la probabilidad de sobrevivir más allá de 1 día es de $88,9 \%$, más de 5 días es $53,3 \%$, y más de 30 días cae a $28,8 \%$ (Figura 1). Se obtuvo dos subgrupos de pacientes de 15 y 8 , respectivamente, de acuerdo a la presencia o ausencia simultánea de los tres reflejos troncoencefálicos: fotomotor, corneal y vestíbulo-ocular. Estos dos subgrupos fueron analizados mediante la curva de Kaplan-Meier con el objeto de poder dirimir si estas variables agrupadas podían actuar como un parámetro predictivo del pronóstico, ya sea en uno u otro sentido (Figura 2). Aunque en el subgrupo caracterizado por la presencia de los 3 reflejos, la posibilidad de una supervivencia mayor a 30 días es de $41,2 \%$ contra $25 \%$ del otro subgrupo, esta diferencia no es estadísticamente significativa de acuerdo al test Log-Rank.

\section{Discusión}

El coma inducido por una injuria anóxica-isquémica cursa en la mayoría de los casos hacia una evolución desfavorable. Al revisar la literatura encontramos que el pronóstico ha sido evaluado en relación a:

I. Estado previo al PCR, como la edad y la enfermedad de base. La edad avanzada, especialmente sobre los 70 años, se relaciona con un mal pronóstico, aunque no tendría valor predictivo por sí sola ${ }^{17}$. La enfermedad de 
base, según algunos autores, no tendría valor predictivo $^{18}$, pero otros investigadores estiman que la diabetes mellitus, la historia de falla cardíaca congestiva, la insuficiencia renal, sepsis, los pacientes quirúrgicos en estado crítico o con falla aguda orgánica, se asocian a un peor pronóstico ${ }^{7,19-21}$.

II. Factores presentes al momento del PCR, como la latencia y duración de las maniobras de resucitación y el lugar del PCR. Una latencia mayor a 5 min en iniciar la resucitación sugiere mal pronóstico ${ }^{19}$. En cuanto al lugar del PCR, si éste se produce en el hospital existiría más posibilidad de sobrevivir que si ocurre fuera de un recinto asistencial ${ }^{22}$. No obstante, algunos estudios sugieren que aquellos pacientes resucitados en una UCI asumen un pésimo pronóstico, debido a la falla multiongánica y sus múltiples complicaciones ${ }^{23}$. En nuestro trabajo, el subgrupo sometido a RCP en el hospital tuvo una mortalidad de $84 \%$ en comparación al 58\% del subgrupo de pacientes resucitados fuera del recinto hospitalario. La duración de las maniobras indica que si el procedimiento de resucitación sobrepasa 15-20 min se correlaciona con peor pronóstico $7,19,24$.

III. Estado posterior al PCR, tales como la evaluación clínica, marcadores bioquímicos, electroencefalografía (EEG), potenciales evocados somatosensitivos (PESS). En lo que respecta a la evaluación clínica, los factores que orientan a un desenlace favorable son la recuperación rápida del coma dentro de las primeras horas y la presencia de reflejos troncales, aunque ningún signo clínico es capaz por sí solo de tener un papel predictivo en forma aislada ${ }^{15}$. Los pacientes que se mantienen en coma después de $48 \mathrm{~h}$ tienen una mortalidad de $77 \%$; cuando el coma se prolonga por más de 3 días, la recuperación es muy rara ${ }^{25,26}$. En cuanto a la recuperación funcional, en el estudio de Levy ${ }^{6}$ solamente 2 de 57 pacientes luego de 3 días en estado comatoso recuperaron función independiente y después de 7 a 14 días, ningún paciente fue capaz de conseguir autonomía. En pacientes con PCR fuera del hospital, la deficiencia en alcanzar el estado de alerta -definido por la presencia de lenguaje comprensible y la obediencia de órdenes- se asocia a una elevada mortalidad y a un período de sobrevivencia menor a 4 días ${ }^{27}$, en cambio, la obtención del alerta es un signo de buen pronóstico y sucede generalmente en los tres días consecutivos al evento ${ }^{28}$. La presencia de lenguaje a las $24 \mathrm{~h}$ es sinónimo de una recuperación completa ${ }^{29}$. Zanderbergen ${ }^{30}$ analizó el resultado precoz en un grupo de 172 pacientes, obtenidos de varios estudios, concluyendo que al cabo de 28 días, $27 \%$ de los pacientes estaba conscientes, $9 \%$ seguía inconsciente y $64 \%$ había fallecido; la posibilidad de sobrevida no se modificaba después de 7 días de la resucitación cardiopulmonar. Estas cifras se asemejan a lo obtenido en nuestro estudio, en que la posibilidad de sobrevida mayor a los 30 días fue de $28,8 \%$ y $75 \%$ de las muertes acontecen a los 7 días. En relación a los reflejos de tronco encefálico, su preservación no necesariamente predice un desenlace favorable. Según Rothstein ${ }^{31}$, 65\% de sus pacientes que conservaban sus reflejos en la evaluación a las $6 \mathrm{~h}$ posteriores al evento anóxico, fallecieron sin lograr despertar. La ausencia de reflejos de tronco encefálico se asocia claramente a un peor pronóstico ${ }^{12,32}$. La ausencia del RFM al momento inicial del examen se asocia a mal pronóstico general ${ }^{6}$, el $90 \%$ de los pacientes sin reflejo corneal a las $24 \mathrm{~h}$ fallece ${ }^{27}$, la abolición del reflejo vestíbulo-ocular se asocia a pronóstico ominoso y en un estudio de pacientes en coma de diversas causas la ausencia combinada de VOR y RFM determinó $100 \%$ de mortalidad $^{2}$. En nuestro trabajo, el análisis de la presencia o ausencia simultánea de los reflejos fotomotor, corneal y vestíbulo-ocular, reveló una tendencia a mayor posibilidad de supervivencia en el subgrupo con preservación de estos reflejos, pero no tuvo significado estadístico. Por otro lado, la ausencia total de estos reflejos no se correlacionó con $100 \%$ de mortalidad al cabo del período de seguimiento.

La presencia de status epiléptico generalizado, tónico-clónico o mioclónico, se asocia a mayor dificultad en la recuperación de la conciencia ${ }^{33,34}$. Al analizar los estudios que han utilizado marcadores bioquímicos como la enolasa neurona específica, $\mathrm{LDH}$, la GOT y la isoenzima creatinkinasa, se ha encontrado que esta última sería de mayor precisión 
pero en términos generales tendría menor valor predictivo que los potenciales evocados ${ }^{35}$. En el EEG, la presencia de coma alfa se asocia en forma frecuente a la encefalopatía anóxica pero no tiene utilidad pronóstica. La ausencia de actividad eléctrica cortical a las $24 \mathrm{~h}$ se asocia a un resultado ominoso pero no logra ser considerada como un elemento predictor categónico ${ }^{36,37}$. La ausencia bilateral de los PESS pronostica muerte sin lograr el despertar entre 73 y $100 \%$, con una especificidad de $100 \%$ a los 7 días $^{12,38}$. La imagenología a través de la RM puede jugar un rol diagnóstico y pronóstico. En un estudio realizado en 10 pacientes usando la técnica de Flair se encontraron alteraciones difusas en la corteza parietal, frontal, tálamo, hipocampo y cerebelo. En ninguno de los pacientes con alteración de señal en la corteza se logró recuperación de mejor calidad que una discapacidad severa ${ }^{39}$.

\section{REFERENCIAS}

1. BeLl JA, Hodgson HJ. Coma after cardiac arrest. Brain 1974; 97: 361-72.

2. Muelier-Jensen A, NeunZig HP, Emskotter T. Outcome prediction in comatose patients: significance of reflex eye movement analysis. J Neurol Neurosurg Psychiatry 1987; 50: 389-92.

3. FANG JF. Prognosis in presumptive hypoxic-ischemic coma in non neurologic trauma. J Trauma Injury Infection 1999; 47: 1122-5.

4. SNyder BD, Ramírez-LasSEPAs M, LipPert DM. Neurologic status and prognosis after cardiopulmonary amest: I. A retrospective study. Neurology 1977; 27: 807.

5. Earnest MP, BreckinRidge JC, Yarnell PR, Oliva PB. Quality of survival after out-of hospital cardiac arrest. Neurology 1979; 29: 56-60.

6. Levy DE, Caronna JJ, Singer BH, Lapinski RH, Frydman H, Plum F. Predicting outcome from hypoxic-ischemic coma. JAMA 1985; 253: 1420-6.

7. Schultz SC, Cuminane DC, Pasquale MD, Magnant C, Evans SR. Predicting in-hospital mortality during cardiopulmonary resuscitation. Resuscitation 1996; 33: 13-7.

8. Denton R, Thomas AN. Cardiopulmonary resuscitation: a retrospective review. Anaesthesia 1997; 52: 324-7.
De nuestro estudio podemos inferir que si el paciente se mantiene en coma luego de $4 \mathrm{~h}$ de la RCP el pronóstico global es malo a corto plazo; que los pacientes con ausencia de los 3 reflejos de tronco tienden a presentar un resultado menos favorable que aquellos con preservación de éstos. Sin embargo, la ausencia total de reflejos de tronco encefálico no se correlaciona con un desenlace fatal en $100 \%$ de los casos al cabo de 30 días, fallando en su utilidad como un elemento absoluto de predicción, al menos en el corto plazo. A pesar de las limitaciones que presenta este trabajo esperamos que pueda servir de orientación o motivación para realizar estudios que contemplen mayor número de pacientes 0 apoyo tecnológico, aunque pensamos que la evaluación clínica sigue siendo lo más relevante a la hora de tomar decisiones.

9. Fertl E, Vass $\mathrm{K}$, Sterz $\mathrm{F}, \mathrm{Gabriel} \mathrm{H}$, Auff E. Neurological rehabilitation of severely disabled cardiac arrest survivors. Part I. Course of postacute inpatient treatment. Resuscitation 2000; 47: 231-9.

10. SHEWmon DA, de Giorgio CM. Early prognosis in anoxic coma: reliability and rationale. Neurol Clin 1989; 7: 823-43.

11. Lee KH, Angus DC, ABRamson NS. Cardiopulmonary resuscitation: what cost to cheat death? Crit Care Med 1996; 12: 2046-52.

12. Zanderbergen EG, de HaAn RJ, Stoutenbeck CP, Koelman JH, Hijdra A. Systematic review of early prediction of poor outcome in anoxic-ischaemic coma. Lancet 1998; 352: 1808-12.

13. Kaplan J. Prediction of poor outcome in anoxicischaemic coma. Clin Neurophysiol 2000; 17: 502.

14. Zoch TW, Desbiens NA, DeStefano F, Stueiand DT, LAYDE PM. Short and long term survival after cardiopulmonary resuscitation. Arch Intern Med 2000; 160: 1969-73.

15. BAtes D. The prognosis of medical coma. J Neurol Neurosurg and Psychiat 2001; 71: 120-5.

16. Teasdale G, Jennet B. Assessment of coma and impaired consciousness: a practical scale. Lancet 1974; 2: 81-4.

17. Murphy DJ, Murray AM, Robinson BE, Campion EW. 
Outcomes of cardiopulmonary in the elderly. Ann Intern Med 1989; 111: 199-205.

18. JoRGENSEN EO. Neurological and circulatory outcomes of cardiopulmonary resuscitation in progress: influence of pre-arrest and arrest factors. Resuscitation 1998; 36: 45-9.

19. Rogove HJ, Safar P, Sutton-Tyrrel K, Abramson NS. Old age does not negate good cerebral outcome after cardiopulmonary resuscitation: analyse from the brain resuscitation clinical trials. The Brain resuscitation Clinical trial I and II Study Groups. Crit Care Med 1995; 23: 18-25.

20. George AL Jr, Folk BP $3^{\text {rd }}$, Creceluus PL, Campbell WB. Pre-arrest morbidity and other correlatos of survival after in-hospital cardiopulmonary arrest. Am J Med 1989; 87: 28-34.

21. Smith DL, Kim K, Cairos BA, Fakhry SM, Meyer AA. Prospective analysis of outcome after cardiopulmonary resuscitation in critically ill surgical patients. J Am Coll Surg 1995; 180: 394-401.

22. Herlitz J, Ekstrom LB, Axelsson A, Holmberg S. Effect of bystander initiated cardiopulmonary resuscitation on ventricular fibrillation and survival after witnessed cardiac arrest outside hospital. Br Heart J 1994; 72: 408-12.

23. Myrianthefs P, Kalafati M, Lemonidou C, Minasidou E, Evagelopoulou P, Karatzas S et al. Efficacy of CPR in a general adult ICU. Resuscitation 2003; 57: 43-8.

24. Sakiayen M, Liss H, Markert R. In-hospital cardiopulmonary resuscitation. Survival in 1 hospital and literature. Medicine (Baltimore) 1995; 74: 163-75.

25. Teres D, BRown RB, LeMEShow S. Predicting mortality of intensive care coma. Crit Care Med 1982; 10: 86.

26. GRuBb NR, Elton RA, Fox KA. In-hospital mortality after out-of-hospital cardiac arrest. Lancet 1995; 346: 417-21.

27. Jorgensen EO, MaLCHOw-Mouer A. Natural history of global and critical brain ischemia. Resuscitation 1981; 9: 133-41.

28. SNyder BD, Loewenson RB, Gumnit RJ, Hauser WA,
LePPIK IE, RAMírEZ-LASSEPAS M. Neurologic prognosis after cardiopulmonary arrest: II. Level of consciousness. Neurology 1980; 30: 52-8.

29. Jorgensen EO, Holm S. Prediction of neurological outcome after cardiopulmonary resuscitation. Resuscitation 1999; 41: 145-52.

30. Zanderbergen EG, de HaAn RJ, Reitsma JB, Hijdra A. Survival and recovery of consciousness in anoxicischemic coma after cardiopulmonary resuscitation. Intensive Care Med 2003; 29: 1911-5.

31. Rothstein TL, Thomas EM, Sum SM. Predicting outcome in hypoxic-ischemic coma. A prospective clinical and electrophysiologic study. Electroencephalogr Clin Neurophysiol 1991; 79: 101-7.

32. SNyder BD, Gumnit RJ, LepPik IE, Hauser WA, LOEWENSON RB, Ramírez-LASSEPAS M. Neurologic prognosis after cardiopulmonary arrest IV. Brainstem reflexes. Neurology 1981; 31: 1092-7.

33. Simon RP, Aminoff MJ. Electrographic status epilepticus in fatal anoxic coma. Ann Neurol 1986; 20: 351-5.

34. Krumholz A, Stern BJ, Weiss HD. Outcome from after cardiopulmonary resuscitation: relation to seizures and myoclonus. Neurology 1988; 38: 401-5.

35. Zingler VC, Krumm B, Bertsch T, Fassbender K, PohLMANN-EDen B. Early prediction of neurological outcome after cardiopulmonary resuscitation. Eur Neurol 2003; 49: 79-84.

36. GuERIT JM. The usefulness of EEG exogenous evoked potentials and cognitive evoked potentials in the acute stage of post-anoxic and traumatic coma. Acta Neurol Belg 2000; 100: 22936.

37. Young GB. The EEG in coma. J Clin Neurophysiol 2000; 17: 473-85.

38. Chen R, Bolton CF, Young B. Prediction of outcome in patients with anoxic coma: a clinical and electrophysiologic study. Crit Med Care 1996; 24: 672 .

39. Wijdicks EF, CAMpeau NG, Miшar GM. MR imaging in comatose survivors of cardiac resuscitation. AJNR Am J Neuroradiol 2001; 22: 1561-5.

Agradecimiento

Los autores expresan su agradecimiento al Dr. Daniel Galdámez P, por sus valiosas sugerencias en el aspecto estadístico. 